\title{
On Application of Graphic Language in Architectural Skin
}

\author{
Honglei Guo and Weimin Guo
}

\begin{abstract}
Visual arts have gone deeply into the life, consumption and the environment of the public after a series of concept innovation such as Postmodernism and Pop Art. The visual effect of graphics is more intuitive and perceptual compared with that of structures, materials and modeling. The visual appeal of the skin should be strengthened by the intuitive, hedonic and current graphic language. The graphic language of the surface delivers construction information and builds visual cognition through the forms such as symbolism, partial decoration, texture pattern, and dynamic image.
\end{abstract}

Index Terms-Graphic language, skin, symbolism, texture, image.

\section{INTRODUCTION}

The skin gradually became independent after experiencing the development of the Modernism and Postmodernism, thus the sense of form of the skin constantly being enhanced, the skin language turning into a more perceptual expression and finally presenting a trend of "Graphical". The aesthetic distance between aesthetic activity and realistic activity has been further shortened after entering the 20th century [1]. Besides the awareness of the public aesthetic consciousness, another incentive for the "Graphical" is the visualized tendency of the information spread in the "the era of picture-reading". People have changed their own way of accepting the information in this society which contains highly developed media. In the current global civilization that considers "the eyes" as the most important organ, when all kinds of social groups tried to identify themselves by using cultural perception and recollection, the graphics has already mastered the "key" to do that [2]. Reading picture directly can not only gain the information with more efficiency, but also obtain vivid experience. In the process of the representational state transfer of the information, the skin reveals some certain kinds of characteristics of public media. People pay less attention to the inherent meaning or the spatial structure of the constructions, and more attentions are paid to seizing and experiencing the exterior visual impression. The graphic language of the surface has become one of the main clues for cognizing and experiencing the construction.

\section{SYMBOLISM}

The changes of social formation, lifestyle and ideas in the post-industrial ages make people issue multiple appeals for

Manuscript received June 10, 2013; revised July 18, 2013.

The authors are with School of Design, Jiangnan University, China (e-mail: guohonglei200@163.com). the value of construction. As a response to the culture default and aesthetics weariness, more and more designers began to pay attention to the expression of the history context and multi-culture, confirm metaphor and symbolism, and make full use of the visual images and symbolic meanings built by the symbols on the surface since postmodernism.

The skin is an information interface made by various kinds of language elements. In the daily expression, information refers to message, intelligence, instructions, and code etc. The information is expressed by some concrete forms such as symbols (characters and words) and signals (language and electromagnetic wave signals) [3]. Graphics are widely used as the symbol of the surface. The signifier and signal of the graphics are easily connected due to the characteristics such as intuition and perception, which offers advantages for the cognition and dissemination. There are some dependent syntax symbols which are made by abundant abstract elements such as shapes, material quality and colors existing on the surface; there are also some independent syntax symbols which are made by many well-defined symbolic graphics existing on the surface. The independent syntax symbols are the main part of the graphic symbols on the skin, which always refer to the figural models, traditional components and specific characters (including language) which are familiar to the public.

The Pop Design which emerged during the 1960s brought popular visual symbols into buildings, product and clothes etc. Architectural images get involved into the modern scenario which contains visual stimulation and language games. For example, The Order as a distinctive classic architectural element, carries with traditional significance, which can express historical feelings. However, in this post-modern cultural revolution which reflected the urban middle class' popularity and rebel consciousness, the Order has already broken the serious and classic tradition and become a kind of popular symbols which are lively, sometimes even joking. The office building of Mazda designed by the Japanese architect Kengo Kuma is a classic example which preserve and reuse the classic architecture vocabulary by using classical order symbols in post-modern era (Fig. 1). This building mixes all the styles from different ages together. It is like a huge ruin which piles all kinds of building components from different times. The Ionic order is the most striking symbol of this building. The identity of the Order changes dramatically. It is evolved from classical building components to the graphical and symbolized skin structures. The overload volume and dramatic role transition bring visual impact and psychological suspense. In post-modern context which contains multi-definitions, the Ionic order with a long history has become a symbol that can stimulate public interest. 


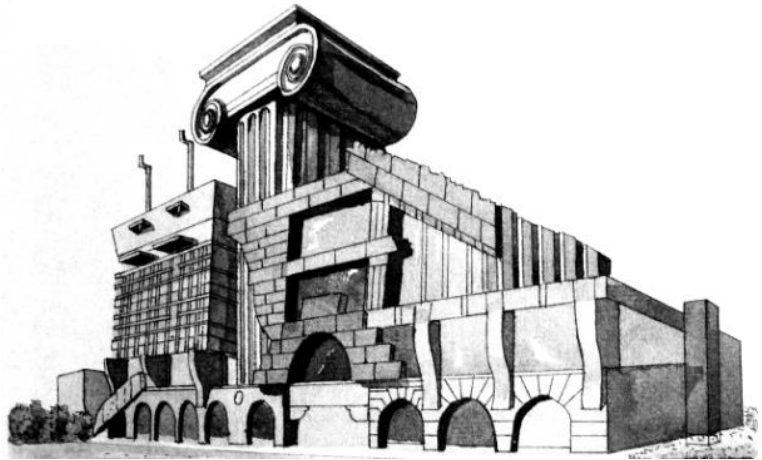

Fig. 1. The symbolism of the Mazda office building surface.

The graphic symbols of the surface can give symbolic expression to the cultural connotation and traditional belief of construction. During the 2010 Shanghai Expo, China Pavilion used bracket set as its symbolism, which delivered abundant and profound spiritual connotation. 24 quadrate reinforced concrete columns slanted outward from the exterior façade of 4 concrete cylinders, forming an upside down half pace. The massive giant pillar rise abruptly out of the ground from the center. Fifty six cross-pieces piled layer after layer, which symbolized the national unity. The symmetrical design contained the golden mean concept. This "Oriental Crown" which was completely built by modern materials and structures embodied the broad and profound Chinese culture through bracket set, the most unique symbol in traditional orders. Korean Pavilion used a unique-designed "Text Skin" as its symbolism, clearly conveying the ethnic and cultural identity. The overall shape of the pavilion is like a huge three dimensional composition that was extended by Korean characters. The whole skin of the pavilion was decorated by Korean words. The façade was bestrewed with delicate embossments and colorful pixel text patterns. With all distance, the image of the letter symbols could be clearly and strongly expressed on the skin (Fig. 2).

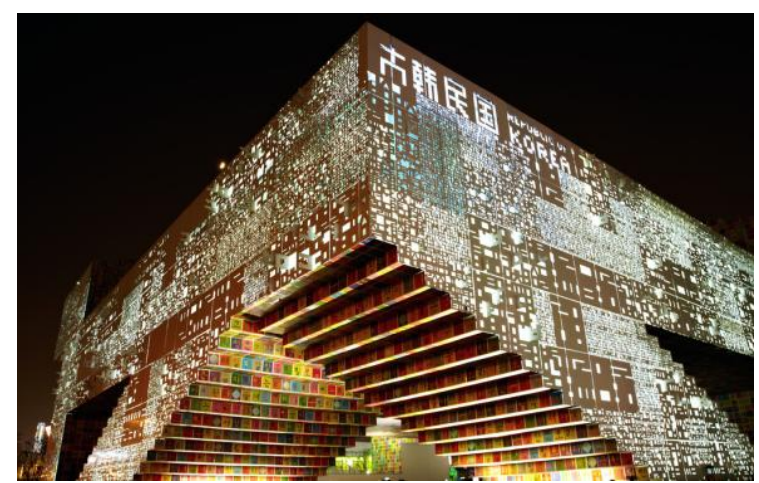

Fig. 2. The letter and graphic symbols on the skin of Korean Pavilion in Shanghai Expo.

\section{PARTIAL DECORATION}

Human beings has begun to use graphics to beautify the surroundings since the period of ancient cave paintings. Ancient Egypt, Ancient Rome and Medieval ruins, and Chinese classical constructions reserve massive architecture decorations with all kinds of forms. The plain or three-dimensional graphic decorations which are made by colored drawing, sculpture, marble and mosaic etc. are widely applied to the façade, ground surface and dome in the western classical architecture. The Baroque architecture carried forward and developed the style of the Renaissance and Medieval period, breaking the limitation between architecture, sculpture and painting and combining them together perfectly. From this, the application of decorative graphics on the exterior architecture façade was pushed to the top. The technique tended to be mature. The graphic decorations were reviewed and promoted on the skin in different degrees in the subsequent neoclassical style, romanticism style, Arts and Crafts Movement, and Art Nouveau.

Under the influence of the dogma that function was always supreme, decorative graphic language was once excluded and rejected by the modern ideological trend of design. Post-modernism lead graphic decoration to its revival. Sheer volume of decorative graphics and words appeared on the surface of buildings, which forms a decorative style called "SuperGraphic Design". The methods of graphic decoration design and building appearance design began to intersect with each other and the languages of them began to be integrated with each other. Postmodern architecture designer Charles Moore cooperated with graphic designer Barbara Solomon, integrating abstract geometrical graphics and fierce colors into skin (Fig. 3). Except for the detail beautified, decorative graphics also have the function of expressing deep implication of space and building environment atmosphere. Decorative elements can not only establish visual connections between different skin elements, but also provide contrasts and differences between all parts of the skin. The decorative skin accords with the public aesthetic demands and modern multicultural context.

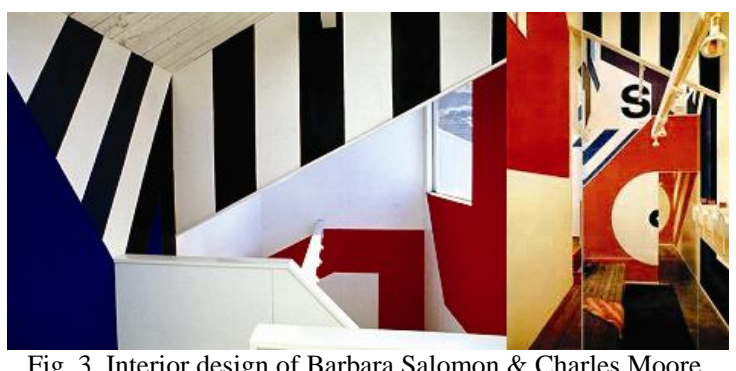

Under the influence of Postmodernism and Pop Art, constructions, products and clothes mix together, reflecting the decorative elements of pop culture. Architectural decorations always adopt the forms which are understandable and with fierce visual stimulation. The urban group which is dominated by the middle class put forward the hedonic and personalized demands from the aspects of consumption and aesthetic appreciation. Popular and lively decoration tactics possess more humanizations and emotions. It makes skin an active stage used for creating entertainment experience. Graves made Walt Disney World Swan own cordial and pleasant expression through the exterior decoration of architecture. A couple of giant swan sculptures were set on the lightly curved top. Two annex buildings in front of the main building were decorated with shell sculptures. The front elevations of both main building and annex buildings were decorated with curved ocean wave pattern. The whole building was located by the lake side, whose decorative elements not only delivered the theme 
vividly, but also blended into the environment. The graphic decorations were filled with rhythms and the sculpture decorations were full of kids' pleasant. Both kinds of decorations brought a unique atmosphere to the Walt Disney World Swan (Fig. 4).

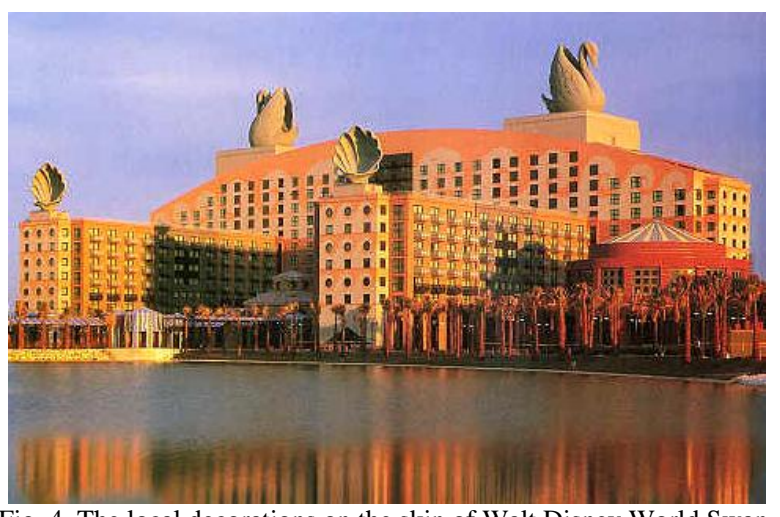

Fig. 4. The local decorations on the skin of Walt Disney World Swan.

In modern architecture, the skin structures can be directly translated into decoration languages. The decorative aesthetic feeling of the skin does not only depend on the add-ons of the outer elements. The Arab World Institute designed by Jean Nouvel set an example for the transition from technical language to decorative language. The cubic sculpt of this construction was quite concise, however, its metal skin possessed quite complicated frames. The skin of the building was divided into two levels. The inside level was transparent wall-curtain made by glass; the outside level was a protective screen made by 27,000 aluminum shutters. The medal protective screen could control the shutters through auto-inductive system and machine driven system. The light adjusting and heat intake could be controlled by the zoom of the aperture. Because the shutter components could open and close freely like iris, this building was honored as "Eyes of the World". Sophisticated parts spliced structured geometrical figure, which formed the Islamic-style window grille with narrow grids, embodying the characteristics of the traditional Arab culture. The metal skin of the Arab World Institute is a perfect example for the mixture of decorative language and technical language (Fig. 5).

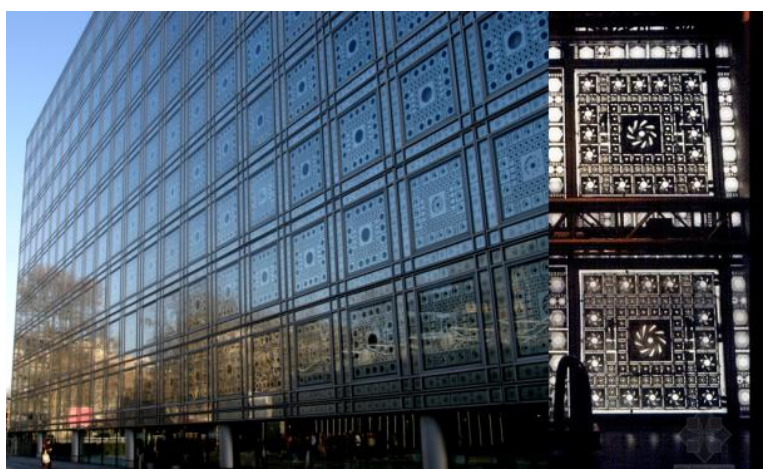

Fig. 5. The façade and detail of the Arab World Institute.

From Romans decorating spaces to Baroque dramatic effects, from Gottfried Semper's clothing theory to Adolf Loos' opposing ornament maxim, people can tell the history of architecture are filled with different opinions on skin decoration. Semper thought that the outer "clothes and ornaments" was more important than the structures, while
Loos considered ornament as a crime, pointing out that the ornamentation had lost its cultural significance in the modern society which stresses sense and efficiency. Robert Venturi and Denise Scott Brown criticized the tedious modernism style ignored the public communication and context continue continuation and put forward that the appearance of architecture should be separated from its function, space, and structure. However, it is hard to touch the public using adoptive decoration symbols which are lacking in common context. Faced with the changing cultural environment and architectural appreciation of the beauty, the decorative language still plays an important role in the skin structures, but the premise is to keep innovating the decoration methods, not just pile up the existing forms. The two giant and curved walls of the Sakura House in Tokyo are made by the $3 \mathrm{~mm}$-wide punching steel plates. The steel walls are covered in Sakura-shaped voids, possessing the traditional Japanese decoration style. The skin of the punching steel plates is processed to white color, making people feel they were in something like a Sakura forest. On one hand, it is isolated from the city noise; on the other hand, it uses façade perforation to establish the connection between inner and outer space. During the night, scattered light shines through the voids, creating a peaceful and romantic atmosphere for the architecture space (Fig. 6).

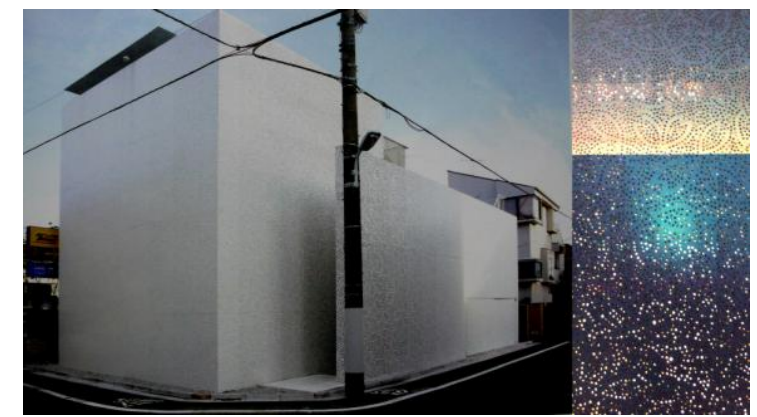

Fig. 6. The skin and detail of the punching steel of the Tokyo Sakura House.

\section{TEXTURE AND PATTERN}

Texture can help skin acquire distinctive visual features. Building novel skin texture has become the current trend of architecture creation. Texture, as a design language which describes the visual attribute of the surface, can not only be directly rooted in the texture of the materials themselves, but also be indirectly formed from the processing and organization forms of the materials. The lightweight skin such as glass curtain wall, metal façade, and cable-membrane structure are not fettered by architectural load-carrying members, which provides great flexibility to the aspect of texture performance. Advanced manufacture techniques and installation processes make those complicated and well-ordered textures appear on the skin. Super computer software such as Maya and Rhino etc. can manipulate the complicated structures, making the skin acquire the texture effect which is quite far beyond reach under normal conditions, and at the same time perfectly realize the function of space maintenance.

Francis Soler applied metal meshes shaped by digital laser cutting technology to the three facades of the Ministry of Culture and Communications (France) building. 
Architects used a special kind of decorative texture which does not contain certain styles to express the inclusive theme of culture and art communication. The free extended texture was hard to perceive its distribution rules, however, some potential regularities could still be sensed. The building of the Ministry of Culture and Communications (France) was located in the traditional rock building groups. The abstract decorative patterns consisted of dynamic lines. The stainless steel skin covered with decorative patterns acted in cooperation with the classical buildings with enriched decorations. The hollowed-out metal skin provided essential safety protections to this government institute. It could guarantee the privacy of the inside space without blocking the vision. Meanwhile, it followed the theme of cultural communication, embodying the characteristics of communicative and open.

The metal skin which was like paper cutting covered the frame structure and glass curtain wall, making the building look more lightsome and transparent. Metal grids, as a kind of strange texture which contains both modernity and tradition, put romantic poetic features and aesthetic images into the architectural skin of this building. This skin texture also decided the visual effect of the inside space. Projections were formed on the floor slabs and walls by sunlight shining through the cutting of the hollowed-out graphics. The landscapes were turned into images full of meanings through the segmentation of the metal grids (Fig. 7).

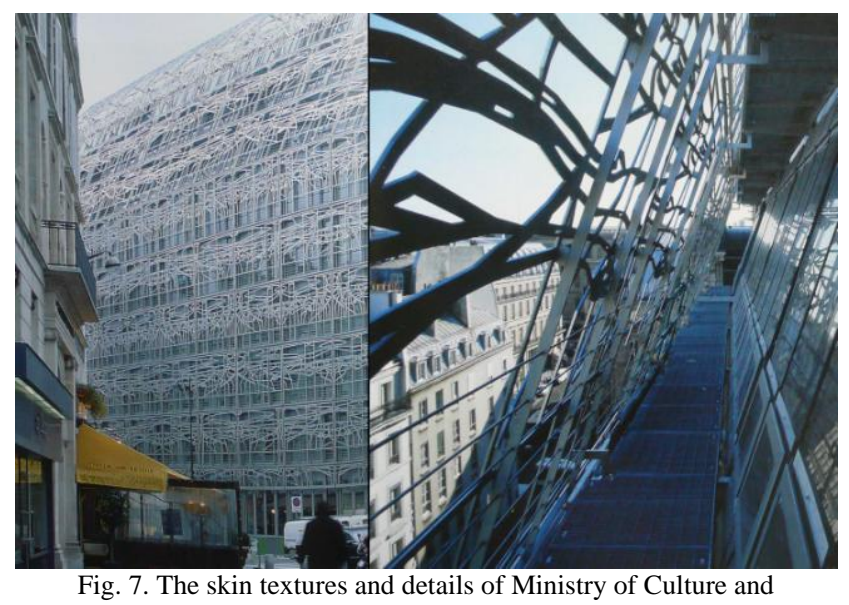

Communication (France) building.

The texture graphics can also be used as fashion elements in the skin. The first hotel of one designer brand called Miss Sixty demanded that the architectural appearance must possess two functions: the first one was it had to be like a beautiful advertisement which could attract the attention of the pedestrians; the second one was it had to show the disposition of easiness and elegance, build fashion preference and deliver brand experience. For these two demands, the façade of the building which was faced to the ocean used some kind of texture graphics similar to the fashion textiles, leading the appearance of the hotel to a young, relaxed, and bright style. There were some elliptic openings covering in the rectangular façade, used as windows. The grids of the openings were kind of hidden, forming organic graphics which are similar to the cheese. The pedestrians will be truly impressed on this giant and clear texture graphic and the fashion style it expresses (Fig. 8)

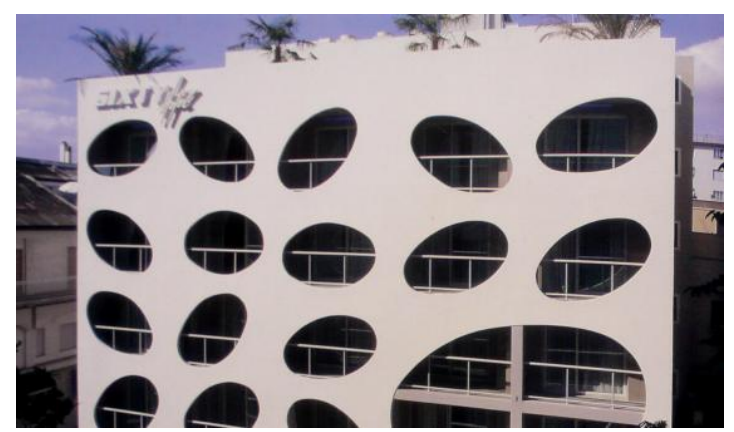

Fig. 8. The skin texture of Miss Sixty Hotel.

The development of the architectural materials provides conditions for the expression of skin texture. Besides the traditional materials such as bricks, glass, concrete, steel, wood, and ceramics etc., in modern architecture skin, synthetic plastics, membrane materials, fabrics or even paper materials are adopted bravely. The constantly changing materials and processing methods gradually enrich the texture materials of the skin. The graphic application of modern architecture skin aims at constructing new images and creating fresh experience, which differs from the post-modern one that borrows historical schematism paste to express the cultural images. By means of constantly developing visual technology, all sorts of non-figurative graphics rooted in new materials gradually form the source of the layout of the skin texture. Herzog \& De Meuron attach importance to the organization of the skin texture, no matter using crusty structure covering the space, or covering the surface with gossamer fabrics, or carving delicate graphics on the surface. The façade of the Ebersealde (A German technology school library) formed a "figured concrete skin" through the gravure. Every unit is an individual graphic. Evenly aligned textures make the appearance of the architecture present unity and orderliness and meanwhile provide excellent local details. The windowpane has the same texture. The only difference is the windowpane use screen printing process to present the translucent effects. The flagship store of Prada in Tokyo adopts the concave and convex glass panels which have diffraction effects. The rhombic-shaped grid texture which contains distortional interiors make the transparent skin shine like diamonds. Swiss Re Headquarters owns a double-deck skin with ventilation system and the spiral textures made by diagrid and dichroic glass. All sorts of graphics made by screens, CNC cutting discs, colored glass, brickwork and facing bricks demonstrate changing textures. These textures exert an enormous influence on the effect of the architecture façade and the vision of the urban environment.

Skin texture can not only create visual effects, but also contain cultural information. The Bradill studio designed by Valerio Olgiati was located in Scharans, Switzerland. The cast pattern of rosettes formed country-style textures on the appearance of brown concrete. "Motif Repeating" is the common method used in traditional furniture decoration. The designers used this method to create skin textures with abstract characteristics, which can not only embody the local processing tradition and country style, but also avoid some over specific imitation. The craftsman in Scharans carved 150 floriated graphics with in different sizes by hand on 60 master-plates which were made by local firs. After 
pouring multiple times, the surface of the concrete demonstrated 550 slightly convex textures, randomly arranged with floriated graphics. The carving marks and the natural texture which the fir master-plates left behind integrate the traditional concept of attaching importance to the manual decoration and material facticity into the hard concrete skin (Fig. 9).

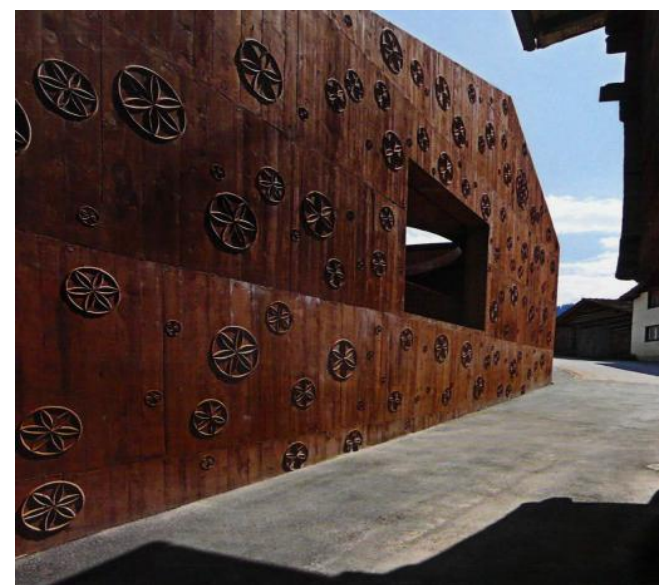

Fig. 9. The graphic textures of the concrete skin of Bardill studio.

\section{DYNAMIC IMAGE}

The visual culture promotes the aestheticization of consumption. With the growth of the visual information and the increase of the visual experience, people are gradually changing their value concept, thinking mode, consumption patterns. The value of consumption changes from the original goods acquisition to significance seeking and aesthetic pleasure. The booming media produce massive visual graphics, gradually changing the way that people receive the information. Digital and media technology enrich the form and style of the graphic language, and exert a great influence on the way of its conveying. Graphic design breaks through the limit of flat dimensions and printing carriers. The media are in the process of further diversification. Dynamic images can do a better job on attracting visual attention, stimulating visual pleasure, satisfying the demands of fast moving consumption. The aesthetic consumption includes environment experience. The image quality of the environment is of great significance in "the society of the spectacle". The dynamic image of the surface can not only provide efficient visual communication, but also render the atmosphere. With the help of the development of communication technology, the skin which can demonstrate dynamic images will become one of the most active factors of the environment in the society of the spectacle.

Before the light-emitting diode (LED) screen came out, the Times Square and Ginza had used large-size televisions forming a big screen to present dynamic images on the buildings. James $\mathrm{P}$ Mitchell and Anaheim invented light-emitting diode display and LED television screen in the end of 1980s. Before long the LED flat-panel display acquired the authentication of American industrial and governmental department, A LCD display technology came out. In 1990s, the technology for high brightness LED display which could completely present RGB colors drove to maturity. This kind of LED display became the media material of dynamic images which was practical for the modern architectural skin, low-cost, and highly efficient. Media technology and digital technology have become the popular vocabulary in the field of modern architectural skin designing. The dynamic image and text information on the light-emitting diode screen concealed the true features of the architecture. The dynamic electronic images which are controlled by the digital terminal became the leading role of the modern urban stage. Skin mediumized is an important way that architecture cope with the complicated social environment. In the book Learning from Las Vegas, Robert Venturi pointed out commercial elements such as advertisement and neon reflected the public preferences. Architects should establish communications with the public and cater to the public interest and values. Electronic cultures were also recreating the aesthetic standards of human beings [4]. The mediumized skin dominated by dynamic images makes architecture develop its manifestation field to a whole new level, which can let architecture still be active in the proscenium of prosperous urban scene.

The mediumized skin of BMW Museum in Munich displays a fascinating "Visual Symphony" by using image technology and interactive installation. The whole surface of the museum is covered with glass panes, 17 million white light-emitting diodes lying behind the panes. The 700 square meters skin is translated into a giant screen which can display dynamic images. The tourists can't realize any existence of technical unit because the dynamic images are displayed on the smooth skin. The dynamic images include not only recorded videos, but also active interaction from all kinds of sensors which can trace the tourists' behavior (Fig. 10).

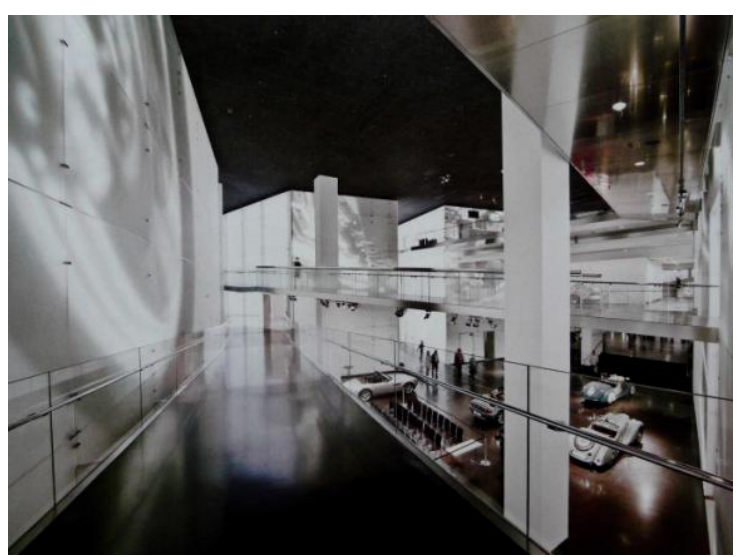

Fig. 10. The dynamic images of the mediumized skin of Bardill and BMW Museum.

The limitation is that, for now, the factors of category and function differences of the architecture were not put into the skin graphics research. The following research includes this exploration of how to apply computer graphics into (skin) surface, based on different architectural categories and functions.

\section{CONCLUSION}

Visual arts have got into the life, consumption and the environment of the public after a series of concept innovation such as Postmodernism and Pop Art. Products, 
properties, and environment are all expressing aesthetic experience and emotional appeal through visual images. Architecture also has an appearance which meets the public aesthetic standards. Skin becomes the making-up of the architecture. It need graphic language to strengthen the infection and endow the architecture with unique charms. The reason is simple-reading images is intuitive, hedonic and current [5]. Business operations and mass media aggravate the trend of "graphical" of the skin. "Graphical" skin shoulders the responsibilities of theme displaying, information orientating, image identifying, brand spreading, environment beautifying, and atmosphere rendering in more and more architectures such as apartments, airports, stations, hospitals, shopping malls, entertainment centers, libraries, exhibition halls, and museums. The graphic language of the surface delivers construction information and builds visual cognition through the forms such as symbolism, local decoration, texture and design, and dynamic image.

\section{REFERENCES}

[1] Z. C. Pan, Edge of the Aesthetic, Shanghai: Shanghai People's Publishing House, pp. 339, 1998.

[2] W. S. Xiao, On Study of Visual Culture and Graphical Awarenes, Beijing: Peking University Press, pp. 16, 2011.

[3] J. H. Ding, Q. Ding, and C. Y. Guo, Cognitive Psychology, Beijing: China Renmin University Press, pp. 4, 2010.

[4] Z. C. Pan, Edge of the Aesthetic, Shanghai: Shanghai People's Publishing House, pp. 129, 1998

[5] X. Zhao, The Veer of Visual Culture, Beijing: Peking University Press, pp. 9, 2008.

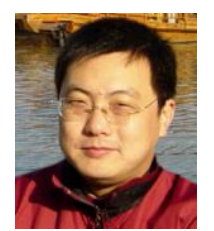

Honglei Guo was born in 1971 in Wuxi. He received his doctor of product design from School of Design, Jiangnan University, Jiangsu Province, China. His Mayor field of study is product and visual communication design.

His previous publication include Corporation and Brand Image Design, Beijing, China, China Building Industry Press, 2010; Graphic Design, Jiangsu, China, Jiangsu Phoenix Press, 2008. His current research focus on computer graphics design and teaching. 